\title{
Off-Diagonal Long-Range Order and Meissner Effect for Lattice Systems
}

\author{
C.-A. Piguet, ${ }^{1}$ D. F. Wang, ${ }^{1}$ and C. Gruber ${ }^{1}$
}

Received June 24, 1996; final January 21, 1997

\begin{abstract}
We study some general properties of a strongly correlated electron system defined on a lattice. Assuming that the system exhibits off-diagonal long-range order, we show that this assumption implies the Meissner effect. This extends to lattice systems previous results obtained for the continuous case.
\end{abstract}

KEY WORDS: ODLRO; Meissner effect; magnetic translations.

It is well known that both the superfluidity and the superconductivity are characterized by existence of off-diagonal long-range orders. For interacting boson systems, Penrose and Onsager have shown that off-diagonal long-range order in the reduced density matrix $\rho_{1}$ is the mathematical criterion for the presence of Bose-Einstein condensation. ${ }^{(1)}$ On the other hand for interacting fermion systems, it was shown by C. N. Yang that superconductivity is characterized by the non-vanishing off-diagonal longrange order in the reduced density matrix $\rho_{2} \cdot{ }^{(2)}$

Two basic features of superconductivity are the Meissner effect and the flux quantization. ${ }^{(3)}$ Therefore if an interacting fermionic system has offdiagonal long-range order, one should be able to deduce the Meissner effect and the flux quantization. In fact, there was some recent proof of Meissner effect as a consequence of existence of ODLRO. ${ }^{(4)}$ Later, this proof was extended to show that the flux quantization also follows from the existence of ODLRO, ${ }^{(5)}$ and to include more general magnetic field distributions. ${ }^{(6)}$ These considerations were concerned with interacting continuous fermion systems mathematically described by Schrödinger

\footnotetext{
'Institut de Physique Théorique, École Polytechnique Fédérale de Lausanne, PHB-Ecublens, CH-1015 Lausanne, Switzerland.
} 
operator. One would expect a similar proof should be true for lattice fermion systems.

Recently, with the discovery of high temperature superconductivity, there have been intense studies on the possibilities of superconductivity of lattice electron systems induced by electron-electron correlations. For the Hubbard model, a notable and the simplest model describing interacting lattice electron system, some $\eta$ pairing states were constructed by Yang in any dimensions. ${ }^{(7)}$ These states have off-diagonal long-range order, and electron systems in these states should be superconducting (unfortunately, these states are not the ground states of the Hubbard model). In the following, we show that the existence of ODLRO for a lattice electron system implies the Meissner effect. This extends the previous results for continuous quantum systems to lattice electron systems.

We consider a system of $N$ interacting electrons in a uniform magnetic field on a two dimensional finite square lattice of size $L \times L$ defined by the two basis vectors $e_{x}$ and $e_{y}$. The Hamiltonian for this system is

$$
H=t_{x} \sum_{i, \sigma}\left(e^{i \theta_{i}^{v}} c_{i+c_{i, .}}^{+} c_{i, \sigma}+\text { h.c. }\right)+t_{y} \sum_{i, \sigma}\left(e^{i \theta \theta_{i}^{i}} c_{i+e_{j, \sigma}}^{+} c_{i, \sigma}+\text { h.c. }\right)+V\left(\left\{n_{i, \sigma}\right\}\right)
$$

where $c_{i, \sigma}^{+}$and $c_{i, \sigma}$ are the creation and annihilation fermionic operators for an electron at site $i$ with spin $\sigma, t_{x}$ and $t_{y}$ are the real hopping amplitudes along the $x$ and the $y$ directions respectively. The phases $\theta_{i}^{x}$ and $\theta_{i}^{y}$ are such that

$$
\theta_{i}^{x}+\theta_{i+e_{x}}^{y}-\theta_{i+e_{y}}^{x}-\theta_{i}^{y}=\frac{e}{\hbar c} \phi
$$

with $\phi$ the magnetic flux through each plaquette of the lattice. The potential $V$ is any real interaction of the electrons, depending only on the numbers of electrons $n_{i, \sigma}=c_{i, \sigma}^{+} c_{i, \sigma}$. Moreover, we impose periodic boundary conditions, i.e., writing $i=\left(i_{x}, i_{y}\right), c_{\left(L+1, i_{y}\right), \sigma}=c_{\left(1, i_{y}\right), \sigma}$ for all $i_{y}$ and all $\sigma$ and $c_{\left(i_{x}, L+1\right), \sigma}=c_{\left(i_{x}, 1\right), \sigma}$ for all $i_{x}$ and all $\sigma$. In the following, assuming existence of ODLRO, we shall show that the magnetic field is necessarily zero.

The Hamiltonian $H$ is not invariant under the usual translations, but it is well known that there exist magnetic translation operators under which the Hamiltonian is invariant both for continuous systems ${ }^{(8)}$ and for lattice systems, if one forgets the boundary terms, i.e., for infinite volume. However, in our case we have to be careful since we are working in a finite volume. For this reason, we take a rational magnetic flux $\phi=(h c / e)(P / Q)$ where $P$ and $Q$ are mutually prime integers $(Q>0)$, and we choose the size of the lattice to be proportional to the magnetic unit cell, i.e., $L=n Q$ where 
$n$ is a positive integer. With this choice and taking for example the Landau

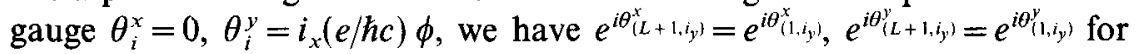

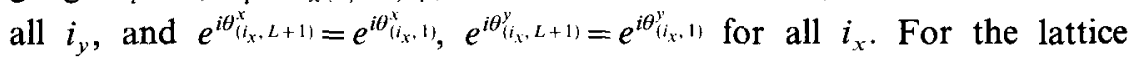
system the two magnetic translation operators $T_{x}$ and $T_{y}$ are defined by the following unitary transformations of the electronic operators

$$
\begin{aligned}
& T_{x}: c_{i, \sigma}^{+} \rightarrow e^{i x_{i}^{x}} c_{i+e_{x}}^{+}, \sigma \\
& T_{y}: c_{i, \sigma}^{+} \rightarrow e^{i x_{i}^{y}} c_{i+e_{y}, \sigma}^{+}
\end{aligned}
$$

where the phases $\chi_{i}^{x}$ and $\chi_{i}^{y}$ are given by

$$
\begin{aligned}
\chi_{i}^{x} & =\frac{e}{\hbar c} i_{y} \phi \\
\chi_{i}^{y} & =0
\end{aligned}
$$

and they satisfy the following relation

$$
\chi_{i}^{x}+\chi_{i+e_{x}}^{y}-\chi_{i+e_{y}}^{x}-\chi_{i}^{y}=-\frac{e}{h c} \phi
$$

Using the fact that the magnetic flux is uniform, it is easy to prove that $T_{x}$ and $T_{y}$ commute with $H$ but not with each other since Eq. (6) gives $e^{i \phi} T_{y} T_{x}=T_{x} T_{y}$. Let us remark that this special choice for the flux and for the size of the lattice is rather technical since it is more convenient to work with a Hamiltonian that has the same periodicity as the one we impose on the system. But this choice will have no physical implications since if we prove that the system cannot support any non zero rational magnetic flux, then the physical meaning will be that the system cannot support any non zero magnetic flux; similarly, when we consider the thermodynamic limit of the system, its size will be irrelevant.

In order to define ODLRO on the lattice, we have to consider

$$
C_{i, j}=\left\langle c_{i, \uparrow}^{+} c_{i, \downarrow}^{+} c_{j, \uparrow} c_{j, \downarrow}\right\rangle
$$

where the brackets is defined by the expectation value in the thermodynamic limit (i.e., letting $L \rightarrow \infty$ and $N / L^{2}$ fixed)

$$
\langle A\rangle=\operatorname{limth} \operatorname{Tr}(\rho(H) A)
$$

where the density matrix $\rho$ is a function of the hamiltonian $H$ (in the following it is not necessary to give an explicit expression for $\rho$ as, for example the Gibbs state given by $\rho(H)=e^{-\beta H} / \operatorname{Tr}^{-\beta H}$, with $\beta$ the inverse 
of the temperature). The trace is performed over the Hilbert space with fixed $N$ number of electrons. The magnetic translations operators allow us to determine how $C_{i, j}$ behaves with respect to translations. Let us choose a basis $\{|n\rangle\}$ of the subspace with $N$ electrons. Since $T_{x}$ is a unitary operator, $\left\{T_{x}|n\rangle\right\}$ is still a basis. We can thus express $C_{i, j}$ in these two different basis

$$
\begin{aligned}
C_{i, j} & =\operatorname{limth} \sum_{n}\left\langle n\left|\rho(H) c_{i, \uparrow}^{+} c_{i, \downarrow}^{+} c_{j, \uparrow} c_{j, \downarrow}\right| n\right\rangle \\
& =\operatorname{limth} \sum_{n}\left\langle n\left|T_{x}^{+} \rho(H) c_{i, \uparrow}^{+} c_{i, \downarrow}^{+} c_{j, \uparrow} c_{j, \downarrow} T_{x}\right| n\right\rangle \\
& =\operatorname{limth} \sum_{n}\left\langle n\left|\rho(H) T_{x}^{+} c_{i, \uparrow}^{+} c_{i, \downarrow}^{+} c_{j, \uparrow} c_{j, \downarrow} T_{x}\right| n\right\rangle \\
& =e^{2 i\left(x_{i}^{*}-x_{j}^{*}\right)} C_{i+e_{x}, j+e_{x}}
\end{aligned}
$$

where we have used the fact that $H$ commute with $T_{x}^{+} l$ and the definition (3) of the magnetic translation operators. With the same manner, we have

$$
C_{i, j}=e^{2 i\left(\chi_{i}^{y}-\chi_{j}^{y}\right)} C_{i+e_{y}, j+e_{y}}
$$

The ODLRO is defined by

$$
\lim _{|i-j| \rightarrow \infty}\left(C_{i, j}-\phi^{*}(i) \phi(j)\right)=0
$$

where $\phi(i)$ is a complex function. Expressing the ODLRO at points $i, j$ and $i+e_{x}, j+e_{x}$ and using Eq. (9), we obtain

$$
\lim _{|i-j| \rightarrow \infty}\left(\phi^{*}(i) \phi(j)-e^{2 i\left(x_{i}^{x}-\chi_{j}^{*}\right)} \phi^{*}\left(i+e_{x}\right) \phi\left(j+e_{x}\right)\right)=0
$$

Since this last equation is true for all $i$ and $j$, we must have

$$
\phi(i)=e^{i \lambda_{x}} e^{2 i x_{i}^{x}} \phi\left(i+e_{x}\right)
$$

where $\lambda_{x}$ is a real number independent of the site $i$. On the other hand, with the help of Eq. (10), we conclude

$$
\phi(i)=e^{i \lambda_{y}} e^{2 i x_{i}^{p}} \phi\left(i+e_{y}\right)
$$

where $\lambda_{y}$ is a fixed real number. 
With Eq. (13) and (14), we can go along the path $i, i+e_{x}, i+e_{x}+e_{y}$, $i+e_{y}, i$ to find a condition on the magnetic flux enclosed by this path:

$$
\begin{aligned}
& \phi(i)=e^{i \lambda_{x}} e^{2 i x_{i}^{x} \phi\left(i+e_{x}\right)} \\
& =e^{i \lambda_{x}} e^{2 i x_{i}^{x}} e^{i \lambda_{y}} e^{2 i x_{i+e_{x}}^{y} \phi\left(i+e_{x}+e_{y}\right)}
\end{aligned}
$$

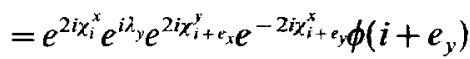

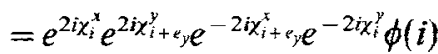

$$
\begin{aligned}
& =e^{-2 i e / h c} \phi(i)
\end{aligned}
$$

where we have used Eq. (6) for the last equality. Equation (15) implies the following constraint on the flux

$$
e^{(2 i e / \hbar c) \phi}=1
$$

and thus the flux must be quantized

$$
\phi=m \frac{h c}{2 e}
$$

with $m$ an integer. If we take $m$ to be 1 , we get a flux which corresponds to a magnetic field of the order of $10^{9} G$, since the area of each plaquette is of the order $\AA^{2}$. Since this value of magnetic field is impossible practically, the only possible choice is $m=0$, which corresponds to zero magnetic field. This is precisely the Meissner effect.

The same argument can be used to discuss the triangular and hexagonal lattices. Let us briefly indicate the main steps for the triangular lattice. The lattice is defined by the two basis vectors $e_{x}$ and $e_{y}$ given in cartesian coordinates by $e_{x}=(1,0)$ and $e_{y}=\left(\frac{1}{2}, \sqrt{3} / 2\right)$. The Hamiltonian has the same form as for the square lattice except that now we have three hopping amplitudes $t_{1}, t_{2}, t_{3}$ and three phase shifts $\theta_{i}^{1}, \theta_{i}^{2}, \theta_{i}^{3}$ corresponding to the three directions $s_{1}, s_{2}, s_{3}$ defined by $s_{1}=e_{x}, s_{2}=e_{y}-e_{x}$ and $s_{3}=-e_{y}$.

In order to define the magnetic translation operators, we choose the specific gauge (with $i=i_{x} e_{x}+i_{y} e_{y}$ )

$$
\theta_{i}^{1}=0, \quad \theta_{i}^{2}=\frac{e}{h c} \phi\left(2 i_{x}-1\right), \quad \theta_{i}^{3}=-\frac{e}{h c} 2 \phi i_{x}
$$

This gauge gives the correct flux $\phi$ across elementary triangles:

$$
\theta_{i}^{1}+\theta_{i+s_{1}}^{2}+\theta_{i+s_{1}+s_{2}}^{3}=\frac{e}{\hbar c} \phi, \quad-\theta_{i+s_{2}+s_{1}}^{3}-\theta_{i+s_{2}}^{\mathrm{r}}-\theta_{i}^{2}=\frac{e}{\hbar c} \phi
$$


In this gauge the magnetic translation operators along the three directions $s_{1}, s_{2}, s_{3}$ are defined by the three phase shifts $\chi_{i}^{1}, \chi_{i}^{2}, \chi_{i}^{3}$

$$
\chi_{i}^{1}=\frac{e}{\hbar c} 2 \phi i_{y}, \quad \chi_{i}^{2}=-\frac{e}{\hbar c} \phi\left(2 i_{y}+1\right), \quad \chi_{i}^{3}=0
$$

Since the flux is uniform, one can easily show that the translation operators defined with such phases commute with the Hamiltonian if we choose the flux to be rational, i.e., $\phi=(h c / e)(P / Q)$ and the size of the lattice to be proportional to the magnetic unit cell, i.e., $L=n Q$. As for the square lattice case, $P$ and $Q$ are mutually prime integers $(Q>0)$ and $n$ is a positive integer. Going around one triangle with the magnetic translation operators, we obtain the opposite of the flux

$$
\chi_{i}^{1}+\chi_{i+s_{1}}^{2}-\chi_{i}^{3}=-\frac{e}{\hbar c} \phi, \quad-\chi_{i+s_{2}+s_{1}}^{3}-\chi_{i+s_{2}}^{1}-\chi_{i}^{2}=-\frac{e}{\hbar c} \phi
$$

The rest of the discussion is similar to the square lattice one and gives the condition that the flux should be quantized

$$
\phi=m \frac{h c}{2 e}
$$

which then leads to the Meissner effect.

In conclusion, we have proved that for a lattice electron system, the assumption of the existence of ODLRO in the ground state (or at finite temperature) implies the Meissner effect. Let us remark that Sewell has mentioned the lattice case but did not give an explicit proof. This remark more explicitly generalizes the previous consideration of continuous electron system described by Schrödinger operator to a correlated lattice electron system. One might wish that for a lattice electron system, the existence of ODLRO would also lead to flux quantization. It would be very interesting to provide a clear mathematical proof of flux quantization as a consequence of existence of ODLRO in a lattice electron system.

\section{ACKNOWLEDGMENT}

This work was supported by the Swiss National Science Foundation.

\section{REFERENCES}

1. O. Penrose, Phil. Mag. 42:1373 (1951); O. Penrose and L. Onsager, Phys. Rev. 104:576 (1956). 
2. C. N. Yang, Rev. Mod. Phys. 34:694 (1962); J. Bardeen, L. N. Cooper, and J. R. Schrieffer, Physical Review 108:1175 (1957).

3. N. Byers and C. N. Yang, Phys. Rev. 7:46 (1961).

4. G. L. Sewell, J. Stat. Phys. 61:415 (1990).

5. H. T. Nieh, G. Su, and Bao-Heng Zhao, Phys. Rev. B 51:3760 (1995).

6. Chi Au and Bao-Heng Zhao, cond-mat/9503024.

7. C. N. Yang, Phys. Rev. Lett. 63:2144 (1989).

8. J. Zak, Phys. Rev. 134:1602 (1964).

Communicated by J. L. Lebowitz 